\title{
Reflets
}

Revue ontaroise d'intervention sociale et communautaire

\section{Incapacité mentale et incapacités sociales}

\section{Louise Blais et Louise Mulligan-Roy}

Volume 4, numéro 2, automne 1998

Personnes vivant avec une incapacité

URI : https://id.erudit.org/iderudit/026219ar

DOI : https://doi.org/10.7202/026219ar

Aller au sommaire du numéro

Éditeur(s)

Reflets : Revue ontaroise d'intervention sociale et communautaire

ISSN

1203-4576 (imprimé)

1712-8498 (numérique)

Découvrir la revue

Citer cet article

Blais, L. \& Mulligan-Roy, L. (1998). Incapacité mentale et incapacités sociales. Reflets, 4(2), 87-103. https://doi.org/10.7202/026219ar

Tous droits réservés (C) Reflets : Revue ontaroise d'intervention sociale et communautaire, 1998
Ce document est protégé par la loi sur le droit d'auteur. L'utilisation des services d'Érudit (y compris la reproduction) est assujettie à sa politique d'utilisation que vous pouvez consulter en ligne.

https://apropos.erudit.org/fr/usagers/politique-dutilisation/ 


\title{
Incapacité mentale et incapacités sociales $^{1}$
}

\author{
Louise Blais et Louise Mulligan-Roy
}

École de service social, Université d'Ottawa

\section{Introduction}

La loi ontarienne sur l'incapacité mentale permet, sous l'avis de spécialistes accrédités, de décider des capacités d'une personne «de prendre soin d'elle-même, de fonctionner dans la collectivité et $[\ldots]$ dans un lieu de travail $»^{2}$. Ce n'est pas notre intention de décrire ou d'analyser cette loi administrative, ni les impératifs qui ont guidé ses récentes modifications, bien qu'il s'agisse là d'un sujet important pour quiconque entreprend de mettre à jour l'histoire du système de psychiatrie et de santé mentale en Ontario (Simmons 1990). Toutefois, dans le contexte de désinstitutionnalisation psychiatrique des dernières décennies, la Loi sur l'incapacité mentale constitue une pièce centrale dans la gestion extra-muros de personnes dont les comportements, les agissements, les croyances, idées ou manières d'être au monde auraient signifié, en d'autres temps et d'autres lieux, l'enfermement à vie en asile psychiatrique. En effet, c'est par le biais de cette loi administrative qu'on se "qualifiera» pour certains programmes d'aide sociale, mais aussi que des contrôles et des traitements seront imposés quand il y aura débordement au niveau des comportements. 
«En effet, il y a quelque chose de l'ordre d'un arrêt de mort sociale que d'être déclaré incapable mental dans une société qui valorise par-dessus tout la performance, le rendement, l'autonomie.»
Cette gestion extra-muros opère à différents niveaux et comprend plusieurs acteurs:

1) le système de santé et le dispositif psychiatrique avec ses institutions hospitalières et communautaires ainsi que ses professionnels dont les psychiatres;

2) le système des services sociaux et le dispositif administratif de l'aide sociale, avec ses divers agents techniques et professionnels; et,

3) le système judiciaire, avec ses lois et les forces de l'ordre.

Ces différents paliers d'examen ayant chacun leurs grilles d'analyse sont autant de pièces à conviction qui contribuent à construire le dossier sur lequel un individu sera déclaré mentalement incapable aux termes de la loi.

Au-delà de ses velléités de protection à la fois de la personne et de la société, la loi sur l'incapacité mentale vient consacrer, et c'est le point de départ de cet article, un statut social dont l'individu ainsi codifié pourra difficilement se relever au cours de sa vie. En effet, il y a quelque chose de l'ordre d'un arrêt de mort sociale que d'être déclaré incapable mental dans une société qui valorise par-dessus tout la performance, le rendement, l'autonomie. Dans les représentations professionnelles et les pratiques institutionnelles, comme dans le «milieu naturel» du reste, que nous la prenions en pitié ou qu'elle nous répugne, l'incapacité mentale rime avec dépendance, déchéance, désordre, danger ${ }^{3}$. Désormais, le moindre geste, la moindre parole seront interprétés et décodés selon une grille de la pathologie, du risque, de l'écart à la norme ou du symptôme et référés à des services trop souvent inappropriés.

Incapable mental, on le deviendra aussi, selon de Certeau (1990), par la perte de légitimité de nos savoirs, connaissances, intelligences dites ordinaires à la base de la vie quotidienne des acteurs sociaux, et ce, à la faveur d'un savoir extérieur, un savoir d'expert qui sait ce qui est bon ou non pour l'autre. Ainsi, le sens que les gens donnent à leurs expériences, leurs besoins et leurs actions a peu de poids. Cela peut même se tourner contre eux dans les divers services si, à plus forte raison, ils sont affublés de l'étiquette d'incapacité mentale, doublée d'un diagnostic 
"L'effort d'adopter le point de vue de l'autre et de comprendre le ou les problèmes à partir de son univers de sens se substitue aux anciennes grilles et catégories pré-établies.» psychiatrique. Et c'est dans cette dynamique de dépossession, si bien décrite par Illich (1976), que se creuse l'écart, voire le malentendu sur la manière d'interpréter et de comprendre les problèmes et les besoins, entre celles et ceux qui prodiguent des services et celles et ceux qui en sont des usagers.

L'idée selon laquelle les services et programmes sociaux sont l'affaire de spécialistes qui décident "pour et à la place de» est de plus en plus questionnée, tant par les mouvements sociaux (groupes de femmes, de défense des droits, de psychiatrisés, etc.) qu'à l'intérieur des diverses disciplines professionnelles qui constatent les limites sérieuses, voire le potentiel iatrogène de leurs interventions. À l'ancienne perspective partant «du haut», on oppose désormais la nécessité d'élaborer des interventions et programmes à partir «du bas», celui de l'univers de sens des gens qui sont les premiers concernés par une situation donnée (Goffman 1968; Bronfenbrenner 1979; Corin et al. 1985; Kleinman 1980, 1988a et b; Tremblay 1997; Blais 1998).

Ceci implique un recadrage de la recherche et de l'intervention dans la manière d'aborder un problème. L'effort d'adopter le point de vue de l'autre et de comprendre le ou les problèmes à partir de son univers de sens se substitue aux anciennes grilles et catégories pré-établies. Les recherches récentes s'inscrivant dans un tel courant, malgré la diversité de leurs objets et de leurs méthodologies (Colin et al. 1992; Bibeau et Perrault 1995; Blais 1993 et 1998; Parent 1995; Corin Rodriguez del Barrio et Guay 1996; St. Amand et Kérisit 1997; Andrew et al. 1997) permettent de faire deux constats. D'abord, ce qui constitue un problème du point de vue des professionnels ne l'est pas nécessairement chez les personnes concernées, et inversement. On est alors confronté au caractère relatif et aux frontières imprécises d'un problème. Ensuite, l'introduction du point de vue des gens «supposément concernés» par un problème ouvre sur les dynamiques socioculturelles qui lui sont sous-jacentes et exposent les conditions cibles à partir desquelles les interventions pourraient être repensées (Bibeau 1986).

C'est sur cette toile de fond que le thème proposé par ce numéro de la revue Reflets a été saisi comme occasion pour faire 
"...on est amené à voir qu'être «incapable mental», aux termes de la loi, n'est pas synonyme d'incapacité à comprendre ce qui nous arrive, de donner sens à notre expérience ou d'élaborer des activités permettant de survive malgré ou avec des difficultés énormes.» une première lecture des données d'une recherche en cours dans la région d'Ottawa-Carleton ${ }^{4}$. Celle-ci se fait, depuis plusieurs années, auprès de femmes et d'hommes psychiatrisés qui sont, par le fait même, les plus susceptibles d'être soumis à la loi sur l'incapacité mentale. En recadrant ainsi la manière d'entendre ce qu'ils ont à dire, on est amené à voir qu'être "incapable mental», aux termes de la loi, n'est pas synonyme d'incapacité à comprendre ce qui nous arrive, de donner sens à notre expérience ou d'élaborer des activités permettant de survivre malgré ou avec des difficultés énormes. Dès lors, on est confronté à des réalités infiniment plus denses et plus complexes, projetant une lumière crue sur des incapacités se situant ailleurs que dans l'individu. L'envers de l'incapacité mentale se révèle alors et pointe vers des incapacités sociales piégeant individus et familles dans ce que Castoriadis-Aulagnier (1981) appelle un "contrat social vicié d'avance».

\section{Psychiatrisation et incapacité mentale, une histoire personnelle et collective}

Sa vie est une histoire de soumissions violentes et de résistances aussi violentes que désespérées. Adoptée avec sa sour jumelle par un couple "dysfonctionnel» (ses mots), elle commencera à avoir des idées suicidaires et des "attaques de panique» (ses mots) dès l'âge de treize ans; $a ̀ 18$ ans elle entre dans un mariage violent qu'elle fuira deux ans plus tard, avec sa vie et les enfants. Elle fera des demandes d'aide auprès des services psychiatriques qui resteront sans réponse, jusqu'à ce qu'elle fasse une première tentative de suicide à 20 ans. Ainsi débutera sa "carrière de folle» ("career as a crazy person» - encore ses mots) avec la première d'un nombre oublié d'hospitalisations sur près de vingt ans, souvent pour des tentatives de suicide graves qui la 
"... «c'est plus dure

que la folie, la

pauvreté, c'est plus

stigmatisant, c'est le

décapage de ton

identité; à partir du

moment que tu es sur

le BS, t'es traitée

comme une menteuse

incompétente et

comme une citoyenne

de deuxième classe

sans droits».» plongent parfois dans des comas. Ces hospitalisations psychiatriques, combinées à une conjoncture d'emplois précaires et faiblement rémunérés, la plongeront à leur tour dans une "carrière de personne pauvre»: (encore ses mots) "c'est plus dure que la folie, la pauvreté, c'est plus stigmatisant, c'est le décapage de ton identité; à partir du moment que tu es sur le BS, t'es traitée comme une menteuse incompétente et comme une citoyenne de deuxième classe sans droits».

Ce récit, que nous traçons à gros traits, ressemble aux récits que nous avons cueillis auprès d'autres personnes dans cette recherche, comme il ressemble à ceux d'autres recherches récentes (Corin, Rodriguez del Barrio et Guay 1996; Blais et Guay 1991; Blais 1998). Ce qui frappe, c'est la récurrence d'un nombre relativement limité de thèmes inscrivant la psychiatrisation et l'incapacité mentale dans un processus et une histoire à la fois personnelle et collective.

Ainsi, il ressort du discours des femmes, une histoire d'abus et de violences qui se déploient sur différents registres et sur un temps long ${ }^{5}$. Sur un premier registre, celui de la famille, l'histoire (les histoires) révèle un enchevêtrement de problèmes à caractère sexuel (la quasi-omniprésence du thème de l'inceste, du viol par les frères, les pères, les grands-pères, les voisins) que l'on taisait, qui n'avait pas droit de parole, de mise en parole avec la mère, les sœurs ou les amies. Puis, suivront des mariages abusifs et d'une violence inouie que l'on subit seule, en silence, jusqu'à la fuite ou la tentative suicidaire qui mène à l'hôpital et à la psychiatrie. À ceux-ci se mêleront des contraintes à caractère culturel dans la mesure où, pour les filles par exemple, la maladie ou la mort précoce d'un des parents impliquaient des responsabilités et des tâches domestiques assumées dès un jeune âge, au point de «ne pas avoir eu d'enfance». Et à travers ces difficultés, se tisseront celles qui sont souvent, mais pas toujours, liées à une pauvreté matérielle extrême qui usent tant le corps physique que le corps psychique surtout quand elles se conjuguent avec d'autres formes d'usure. 
Sur un second registre, celui des pratiques professionnelles et institutionnelles, si les récits témoignent parfois de ce qu'il peut y avoir de positif, d'aidant ou de constructif dans l'intervention, le plus souvent, ils projettent une lumière crue sur une pratique professionnalo-technique et institutionnelle incroyablement détachée, indifférente, aseptisée. Ainsi, à la première interdiction de mise en parole dans le milieu familial, les récits accusent les milieux d'intervention, dont la psychiatrie, d'en imposer une deuxième en empêchant qu'un discours ne s'élabore sur l'histoire personnelle remplie de difficultés, parfois extrêmes, telles la pauvreté, les deuils précoces, les abus sexuels et physiques vécus dans l'enfance ou comme adulte. Plusieurs diront: "Ils [les intervenants] ramenaient toujours les problèmes à moi», pour manifester cette seconde interdiction de mise en parole. Comment ce réel-là a-t-il joué, et joue encore, dans la détresse? Comment a-t-il façonné la vie, l'identité, le rapport à soi et à autrui?

Ces données doivent être mises en relation avec les mises en garde venant d'horizons divers contre des pratiques professionnelles et institutionnelles qui ne tiennent pas compte de l'histoire personnelle et du contexte dans lequel s'inscrivent les problèmes des gens (Kleinman et Kleinman 1997; Blais 1998).

\section{Misères quotidiennes et fragilité du lien social}

Si les récits révèlent les éléments de contexte de vie qui ont graduellement mené à la psychiatrie et, de fil en aiguille, au statut d'incapable mental, ils révèlent aussi l'impact de la psychiatrisation sur l'identité et la trajectoire sociales des personnes concernées. En d'autres termes, il y aura eu un avant et un après, ce qui a conduit à la psychiatrie et ce qui en a découlé. De fait, ces récits font ressortir les nombreuses contraintes qui traversent la vie des personnes affublées des étiquettes d'incapacité mentale et de psychiatrisé. Ils mettent en lumière une situation de pauvreté qui affecte profondément leur équilibre au quotidien et qui conjugue souvent misère matérielle et fragilité du lien social. En effet, nos 
En effet, nos données refletent celles

rapportées dans un nombre grandissant de recherches récentes... montrant comment les personnes psychiatrisées constituent, avec d'autres, tels les sidéens, les sans domicile fixe, les sans papiers, etc., les figures contemporaines de l'exclusion.

"Une femme interrogée nous dira : «Sans logement adéquat, rien ne peut se stabiliser. On a besoin de sécurité pour être stable».» données reflètent celles rapportées dans un nombre grandissant de recherches récentes (Blais et Guay 1991; de Gaulejac et Taboada Léonetti 1994; Capponi 1992; Robichaud et al. 1994) montrant comment les personnes psychiatrisées constituent, avec d'autres, tels les sidéens, les sans domicile fixe, les sans papiers, etc., les figures contemporaines de l'exclusion.

Le discours des personnes interrogées sur leurs conditions d'existence témoigne de détresse et d'angoisse vécues quotidiennement. D'où les préoccupations constantes liées au manque chronique d'argent, la frustration continue des besoins et des désirs, le drame de la faim des fins de mois, l'organisation de la vie autour d'activités et de démarches qui visent à combler les besoins vitaux, l'interdiction de "petits luxes», la frustration quotidienne de ne pas se sentir "comme les autres». De surcroit, le logement, particulièrement son état et son coût, est souvent une source importante d'instabilité et d'insécurité qui vient fragiliser une situation déjà très précaire. Une femme interrogée nous dira : «Sans logement adéquat, rien ne peut se stabiliser. On a besoin de sécurité pour être stable». Ces personnes dont les ressources proviennent principalement de l'assistance sociale ont peu de chances de pouvoir habiter un logement décent sur le marché privé et l'accès aux logements sociaux demeure extrêmement limité.

Il est important de signaler que plus des trois-quarts des personnes interrogées disposent d'un revenu annuel inférieur à 11000 \$, revenu qui se trouve bien en deçà du seuil de faible revenu établi à 16609 \$ par Statistiques Canada pour un individu demeurant seul dans une région comptant de plus de 500000 personnes, comme Ottawa-Carleton. Selon Statistiques Canada, un ménage devant consacrer $55 \%$ ou plus de son revenu à la satisfaction des besoins essentiels (logement, nourriture et vêtements) vit sous le seuil de la pauvreté alors que ce seuil se situe à $50 \%$ pour le Conseil canadien de développement social (Ross et al. 1994:16-19). Pour les personnes interrogées bénéficiant d'un logement subventionné, leur loyer s'élève à 106 \$ en moyenne par mois, ce qui représente $19 \%$ du revenu allant au logement. En revanche, les personnes louant un logement sur le marché 
privé, ce chiffre grimpe à $479 \$$, soit près de $50 \%$ de leur revenu. Dans ce dernier cas, lorsque les ressources sont versées pour répondre aux autres besoins vitaux, il ne reste pratiquement rien ou presque à consacrer aux frais de transport, aux soins de santé, aux soins personnels et aux loisirs.

Le problème de la pauvreté ne se limite pas à la dureté «objective» des conditions d'existence. Il comprend également les répercussions «subjectives» sur la psyché (de Gaulejac 1996). La pauvreté peut occasionner une souffrance psychique particulièrement douloureuse pour les personnes vivant dans cette situation, souffrance qui va bien au-delà de la privation matérielle pour rejoindre des dimensions qui touche l'ordre du social et du symbolique (de Gaulejac 1987; Colin et al. 1992; Blais 1998). Vivre quotidiennement en situation de pauvreté entraîne des malaises de l'âme que les personnes expriment de diverses manières: honte, dévalorisation, colère, révolte, désespoir, humiliation, rejet de la part de l'entourage et de la société plus large. Ils demeurent longuement gravés dans la psyché.

Aussi, les témoignages révèlent-ils un vécu quotidien empreint de souffrance qui est intimement liée au regard de la société qui juge, condamne et humilie (de Gaulejac 1996), mais aussi à la conscience de la place qu'on occupe ou qu'on n'occupe pas dans la société :

J'ai tellement été habitué, avant de connaître mes psychoses, à être bien actif dans la société, valorisé aussi. J'avais des rôles valorisants puis un statut valorisé aussi, soit travailleur, soit étudiant, ou soit les deux en même temps, sportif puis tout ça. Le vide que je ressens, c'est le rejet social que je ressens. Je vis dans un ghetto de psychiatrisés avec quelques amis qui ne sont pas psychiatrisés mais qui sont sur l'aide sociale.

Ces personnes affublées des étiquettes d'incapacité mentale et de psychiatrisé décrivent, d'une manière ou d'une autre, les processus de mise à l'écart, de marginalisation, d'invalidation sociale et de stigmatisation. Ceux-ci les renvoient toujours à leur différence et finissent par se traduire en blessures qui marqueront profondément 
leur identité. En effet, en plus d'isoler les personnes concernées, cette différence leur renvoie leur manque, leur incompétence, leur «inadaptation» sociale.

De plus, les récits font écho à un sentiment de rejet, de honte et d'impuissance dans le rapport aux institutions chargées d'assister les individus en difficulté et qui viennent souvent mettre à rude épreuve l'équilibre personnel. Soulignant qu'ils étaient «obligés» de demander de l'aide, geste dont nous avons montré ailleurs le caractère complexe (Blais 1998), et qu'il s'agissait là pour eux d'une question de survie, l'assistance sociale est souvent vécue comme une humiliation. Les individus reconnaissent la nécessité de l'assistance, mais expriment une colère et une souffrance d'être obligés de se soumettre à une série de conditions et de procédures qu'ils vivent comme dévalorisantes :

C'est plus dure que la folie, la pauvreté. C'est plus stigmatisant, c'est le décapage de ton identité; à partir du moment que tu es sur le bien-être social, t'es traitée comme une menteuse, une incompétente et comme une citoyenne de deuxième classe, sans droits. En même temps, t'es sujet à un système où, en dépit de qu'est-ce qu'on te dit, t'as pas de droits [...] Je savais ce que ce serait d'être pauvre et de vivre de l'aide sociale. Dans mon esprit,j'étais mieux d'être morte que de passer à travers cette humiliation [...] J'ai fait une tentative de suicide.

À maintes reprises, les récits dénoncent l'étiquetage du dispositif psychiatrique qui, si souvent, fige la personne dans un statut auquel elle pourra difficilement échapper :

Pendant des années, jusqu'à temps que je vienne à bout de faire fermer le dossier, la minute que j'étais malade physiquement, en entrant à l'urgence, automatiquement, ils m'envoyaient en psychiatrie. Toutes les portes sont fermées aux psychiatrisés. Au niveau de l'emploi, quand tu dis à une personne que t'es psychiatrique, à un employeur, mettons sur une 
"La marginalité et l'exclusion seraient l'aboutissement d'un double processus de décrochage qui peut se lire sur les axes de l'insertion professionnelle et de l'insertion relationnelle.» application pour avoir un emploi, à moins que ça soit un emploi protégé, dans le sens qu'ils ont des subventions pour engager la personne qui a des problèmes en psychiatrie... des préjugés circulent dans la société contre les psychiatrisés.

Le rejet, le refoulement vers la marge, la stigmatisation, la fragilité des liens sociaux qui peut aller jusqu'à compromettre la citoyenneté, imposent aux personnes une image négative d'ellesmêmes, celle d'un citoyen sans utilité sociale, sans raison d'être (Roy 1995; Debordeaux 1994). «[C]'est le regard décapant avec lequel cette même société les observe qui empêche de déceler leurs potentialités, leurs aspirations, leurs contributions à la vie collective», diront Robichaud et al. (1994 : 23).

Castel $(1995 ; 1994)$ dirait qu'il faut voir dans cette situation de marginalité et d'exclusion, une réalité qui traverse la société contemporaine dans son ensemble. La marginalité et l'exclusion seraient l'aboutissement d'un double processus de décrochage qui peut se lire sur les axes de l'insertion professionnelle et de l'insertion relationnelle. Ces deux axes permettent de définir, selon le degré d'intégration, trois zones: la zone d'intégration, la zone de vulnérabilité et la zone de désaffiliation. Être dans la zone d'intégration signifie que l'individu dispose des garanties d'un emploi stable et qu'il bénéficie d'une insertion relationnelle solide. La zone de vulnérabilité est synonyme de précarité d'emploi et de fragilité relationnelle. Enfin, la zone de désaffiliation associe l'absence d'emploi et l'isolement social. La désaffiliation désigne un double décrochage au terme duquel la «précarité économique est devenue dénuement, la fragilité relationnelle isolement» (Debordeaux 1994: 94). Être dans la zone de désaffiliation, c'est vivre une rupture de l'appartenance, voire une rupture du lien sociétal.

Les zones décrites ne sont pas figées, puisque leurs frontières sont poreuses. Il s'effectue constamment des transitions de l'une à l'autre, en particulier de la zone de vulnérabilité vers la zone de désaffiliation. La zone de vulnérabilité occupe une position stratégique, car elle constitue un espace social de turbulences et 
d'instabilité composé de personnes dont le rapport à l'emploi est précaire et dont l'inscription dans des réseaux de sociabilité est fragile. Cette zone correspond à une fragilité du lien social avant sa rupture, d'où le risque de basculement dans la zone de désaffiliation.

À l'instar de Castel, nous pensons que les personnes que nous avons interrogées sont, à divers moments, dans la zone turbulente et instable de la vulnérabilité et ont parfois, faute d'une intervention appropriée, basculé dans la désaffiliation.

\section{Stratégies au quotidien}

"Le statut d'incapacité mentale et de psychiatrisé signifie trop souvent, dans les milieux d'intervention formels et institutionnels, des personnes dépendantes et incapables de prendre soin d'ellesmêmes.»

"Ils sont capables d'initiatives et d'actions, de pression et de résistance qui leur permettent de maintenir, de retrouver ou de réinventer un équilibre.»
Le statut d'incapacité mentale et de psychiatrisé signifie trop souvent, dans les milieux d'intervention formels et institutionnels, des personnes dépendantes et incapables de prendre soin d'ellesmêmes. Nos données préliminaires révèlent un tableau infiniment plus complexe.Jusqu'ici notre lecture des données a mis en lumière les contraintes, parfois extrêmes, émanant du contexte pouvant plonger les individus dans les zones de vulnérabilité, voire de désaffiliation. Cela dit, nous avons aussi cherché à voir les stratégies de vie et de survie, comprises ici comme un ensemble de pratiques ou d'actions que les gens mettent en branle pour gérer les contraintes du quotidien et trouver une marge de manœuvre. Dit autrement, si les histoires des gens les révèlent comme des sujets assujettis à des contraintes et des difficultés inouïes, elles les révèlent, dans un même souffle, comme des sujets résistants, capables à la fois de donner sens à leurs expériences aussi bien que d'élaborer des actions pour pouvoir vivre au jour le jour. Leurs témoignages obligent à reconnaître qu'ils disposent de ressources individuelles et collectives. Ils sont capables d'initiatives et d'actions, de pression et de résistance qui leur permettent de maintenir, de retrouver ou de réinventer un équilibre.

Il importe de souligner qu'en faisant place aux stratégies élaborées par des personnes lourdement hypothéquées sur le plan personnel et social, notre objectif ne consiste pas à occulter leur 
dure réalité, et faire comme si tout allait pour le mieux dans le meilleur des mondes. Ce serait là, en effet, donner inconsciemment raison aux tenants du néolibéralisme et leurs politiques de «déficit zéro». Bien au contraire, l'intention derrière notre pratique de recherche est de faire valoir les initiatives prises par les plus fragiles et les plus fragilisés de notre société pour se tailler des conditions de survie et des espaces de liberté qui pourraient servir de point de départ pour redéfinir des pratiques d'intervention qui soient mieux arrimées à leurs besoins.

Les récits nous permettent de saisir comment les personnes psychiatrisées se meuvent dans la cité, utilisent les diverses ressources dans la communauté afin de faire face à la situation de pauvreté et de stigmatisation qui pèse sur elles et menace sans cesse un équilibre déjà fragile. Ainsi, les gens ont recours de façon régulière aux soupes populaires, souvent organisées par les églises protestantes ou catholiques, aux comptoirs de vêtements, aux banques alimentaires pour combler leurs besoins vitaux que le filet de sécurité des prestations d'assistance sociale n'arrivent plus à combler.

Mais ce sont les regroupements de type associatif ayant émergé au cours des vingt dernières années de désinstitutionnalisation psychiatrique qui occupent une place particulièrement importante dans les stratégies de survie de ces personnes. Groupes d'entraide, de défense des droits, haltes accueil et autres, ces regroupements s'inscrivent au coeur d'un réseau relationnel de quartier. Peu nombreux et disposant de maigres ressources matérielles, ces regroupements contribuent néanmoins à renforcer les solidarités en offrant aux individus la possibilité de recréer des liens sociaux fondés sur l'échange et la réciprocité, générant ainsi une reconnaissance symbolique qui sert d'appui à la construction d'une identité personnelle positive. Aussi, ces regroupements semblent servir de rempart contre les sentiments d'insécurité, de marginalisation, de stigmatisation qui hantent si souvent les personnes affublées des étiquettes d'incapacité mentale et de psychiatrisé. Quoique le niveau d'implication varie d'une personne à l'autre, elles définiront ces regroupements comme des lieux de 
répit, d'inclusion et de reconnaissance où elles se sentent accueillies, respectées, acceptées et valorisées :

C'est en aidant les autres qu'on s'aide soi-même [...]

On s'explique sans parler. On n'est pas obligé de passer par mille chemins. On a un vécu psychiatrique et on a $v u$ comment les autres ont réagi à ça... d'autres amis qui n'étaient pas des vrais amis. Tu réalises qu'il y a $d u$ bon monde là dedans. C'est que ça apporte un certain confort, une compréhension. Comme ici, c'est un petit lieu de recueil.

Quand je suis triste, j'ai du monde à qui je peux le dire, du monde qui vont avoir de la compassion. La sympathie, la compassion, l'amitié, la fraternité, l'égalité... parce qu'on a une souffrance commune [...].

\section{En guise de conclusion}

Cette étude, comme celles dont nous avons fait mention ci-dessus, nous forcent à revoir nos représentations des gens étiquetés du statut d'incapacité mentale. En effet, les récits montrent de longues histoires individuelles où s'enchevêtrent de multiples contraintes, souvent contradictoires, émanant d'abord du milieu familial, pour se prolonger par la suite dans les divers milieux de la société : l'école, le travail, les services sociaux et de la santé, notamment la psychiatrie.

L'incapacité mentale se révèle alors comme une sorte d'écran de fumée venant occulter des incapacités au niveau social et collectif. Qu'il s'agisse des abus sexuels ou physiques, des privations extrêmes et chroniques dues à la pauvreté ou encore des comportements jugés hors normes, par exemple l'hyperactivité, les récits mettent en lumière une souffrance individuelle qui a toujours sa source dans un contexte collectif et social, mais qui n'a pas pu trouver une résonance, c'est-à-dire une reconnaissance 
hors soi. De là provient la dérive vers la tentative de suicide, la dépression, la psychose, la délinquance, et surtout leur cristallisation dans la chronicité.

Or, la souffrance n'a pas bonne presse dans les milieux d'intervention où l'on préfere les catégories plus propres et plus aseptisées des manuels diagnostics. Pourtant, comme l'ont remarqué Das, Cavell et Kleinman, devant l'expression de la souffrance, on est toujours appelé à répondre, à réagir, à choisir, soit en la reconnaissant, soit en l'évitant. Ne pas répondre serait nier son existence, ce qui est en soi un acte de violence, un acte qui perpétue la violence initiale. De là découle l'importance, voire l'exigence éthique et sociale, de symboliser la souffrance avec des mots qui ont une résonance et un sens autrement plus riches que ceux des catégories diagnostiques du lexique "psy». L'enjeu, en somme, consiste à se donner collectivement des symboles évocateurs ayant un pouvoir susceptible d'engager la société, les milieux d'intervention y compris, dans un processus autre que celui de mise à l'écart de personnes vulnérables, comme c'est le cas avec le statut d'incapacité mentale.

\section{Bibliographie}

ANDREW, C. ET AL. (1997). Les conditions de possibilité des services de santé et des services sociaux en français en Ontario: un enjeu pour les femmes, Ottawa, Table féministe francophone de concertation provinciale de l'Ontario.

BIBEAU, G. (1986). «Le facteur humain en politique: application au domaine de la santé mentale», Santé mentale au Québec, vol. 11, no. 1,19-41.

BLAIS, L. (1998). Pauvreté et santé mentale au féminin. L'étrangère à nos portes, Ottawa, Presses de l'Université d'Ottawa.

BLAIS, L. ET L. GUAY (1991). «Pauvreté, santé mentale et stratégies d'existence», Santé mentale au Québec, vol. 16, no. 1,117-138.

CAPPONI, P. (1992). Upstairs in the Crazy House, Toronto, Penguin Books.

CASTEL, R. (1994). «La dynamique des processus de marginalisation: de la vulnérabilité à la désaffiliation", Cahiers de recherche sociologique, no. 22, 12-27.

CASTEL, R. (1995). Les métamorphoses de la question sociale. Une chronique du salariat, Paris, Fayard.

CASTORIADIS-AULAGNIER, P. (1981). La violence de l'interprétation: du pictogramme à l'énoncé, Paris, P.U.F., coll. le fil rouge.

CAVELL, S. (1997). "Comments on Veena Das's Essay "Language and Body: Transactions in the Construction of Pain"”, dans Social Suffering, sous la dir. de A. Kleinman, V. Das et M. Lock. Berkeley, University of California Press, 93-98. 
CERTEAU, M. DE (1990). L'invention du quotidien, Paris, Gallimard.

COLIN, C., F. OUELLET, BOYER ET C. MARTIN (1992). Extrême pauvreté, maternité et santé, Montréal, Éditions Saint-Martin.

CORIN, E., L. RODRIGUEZ DEL BAARRIOET L. GUAY (1996). «Les figures de l'aliénation: un regard alternatif sur l'appropriation du pouvoir», Revue canadienne de santé mentale, vol. 15, no. 2, 45-67.

CORIN, E., F. GRUNDBER G G. SÉGUIN-TREMBLAY ET L.TESSIER (1985). La santé mentale: de la biologie à la culture. Avis sur la notion de santé mentale, Québec, Ministère de la Santé et des Services sociaux. Comité de santé mentale du Québec.

DAS,V. (1997). «Language and Body:Transactions in the Construction of Pain», dans Social Suffering, sous la dir. de A. Kleinman,V. Das et M. Lock. Berkeley, University of California Press, 67-91.

DEBORDEAUX, D. (1994). «Désaffiliation, disqualification, désinsertion», Recherches et Prévisions, no. 38, 93-100.

DEVREUX, A.-M. (1986). Biographies masculines et féminines et mémoire sexuée : l'exemple des modèles parentaux. Communication au XIe Congrès Mondial de Sociologie. New Dehli, 18-24 août.

GAULEJAC,V. DE (1987). La névrose de classe, Paris, Hommes et Groupes Éditeurs.

GAULEJAC,V. DE (1996). Les sources de la honte, Paris, Desclée de Brouwer.

GAULEJAC,V.DE ET I.TABOADA LÉONETTI (1994). La lutte des places. Insertion et désinsertion, Paris, Desclée de Brouwer.

GOFFMAN, E. (1968). Asiles. Études sur la condition sociale des malades mentaux, Paris, Éditions du minuit.

ILLICH, I. (1976). Némésis médical, l'expropriation de la santé, Paris, Seuil.

KLEINMAN,A. (1980). Patients, Healers and the Context of Culture, Berkeley, University of California Press.

KLEINMAN,A. (1988a). The Illness Narratives; Suffering, Healing and the Human Condition, New York, Basic Books.

KLEINMAN, A. (1988b). Rethinking Psychiatry: From Cultural Category to Personal Experience, New York, Free Press.

KLEINMAN, A. ET J. KLEINMAN (1997). «The Appeal of Experience ; The Dismay of Images : Cultural Appropriations of Suffering in ourTimes», dans Social Suffering, sous la dir. de A. Kleinman, V. Das et M. Lock. Berkeley, University of California Press, 1-23.

ONTARIO (1998). Loi de 1997 sur le Programme ontarien de soutien aux personnes handicapées. Lois de l'Ontario de 1997, Chapitre 25, Annexe B.

PARENT, C. (1995). «La “prostitution” ou le commerce des services sexuels», dans Traité des problèmes sociaux, sous la dir. de F. Dumont, S. Langlois et Y. Martin. Québec, IQRC, 393-409.

ROBICHAUC, J.-B., L. GUAY, C. COLIN, M. POTHIER ET J.-F. SAUCIER (1994). Les liens entre la pauvreté et la santé mentale, Montréal, Gaëtan Morin éditeur.

ROSS, D. P., E. R. SHILLINGTON ET C. LOCHHEAD (1994). The Canadian Fact Book on Poverty, Ottawa, Conseil canadien de développement social.

ROY, S. (1995). «L'itinérance: forme exemplaire d'exclusion sociale?», RIAC, vol. 74, no. 34, 73-80. SIMMONS, H. G. (1990). Unbalanced: Mental Health Policy in Ontario, 1930-1989, Toronto, Thompson Educational Publishing. 
ST-AMAND, N.ET M. KÉRISIT (1997). «Résistance et créativité : pratiques alternatives des familles démunies", Reflets. Revue ontaroise d'intervention sociale et communautaire, vol. 3, no. 1, 30-54.

TREMBLAY, M.A. (1997). «Préface» dans La recherche qualitative : les enjeux épistémologiques et méthodologiques, sous la dir. de J. Poupart, J. P. Deslauriers, L. Groulx,A. Laperrière, R. Mayer et A. Pires. Montréal, Gaëtan Morin éditeur, xvii-xxxvii.

\section{Notes}

1. Le matériel sur lequel se base cet article fait partie d'une recherche plus large que nous menons portant sur la spécificité des discours et pratiques alternatifs dans le champ de la santé mentale en Ontario. La recherche s'inscrit dans les travaux de l'Équipe de recherche et action en santé mentale et culture (Érasme). Cette équipe, financée par le Conseil québécois de la recherche sociale (CQRS) (subvention no. RS2821M96) et dirigée par Ellen Corin, est constituée de chercheurs universitaires et de groupes communautaires. Les partenaires universitaires sont: Gilles Bibeau (Université de Montréal, anthropologie); Louise Blais (Université d'Ottawa, service social); Ellen Corin (Université McGill, psychiatrie et anthropologie); Jocelyne Lamoureux (UQAM, sociologie); Cécile Rousseau (Université McGill, psychiatrie). Les partenaires communautaires sont: l'Institut interculturel de Montréal (Kalpana Das, Robert Vachon, Marc Perreault et Émongo Lomomba); le Regroupement des ressources alternatives en santé mentale du Québec (Lorraine Guay et Lourdes Rodriguez del Barrio); la Table de concertation des organismes de Montréal pour les réfugiés (Stéphane Reichhold, Abdelwahed Mêkki-Berrada et Marie-Claire Rufagari). La recherche a également bénéficié de diverses subventions de la Faculté des sciences sociales et de l'École des études supérieures et de la recherche de l'Université d'Ottawa.

2. Loi de 1997 sur le Programme ontarien de soutien aux personnes handicapées, Partie 1 article 4.1.b.

3. Le terme anglais employé en Ontario est encore plus fort: «mental incompetence». 
4. L'étude dont il est question cherche à montrer ce qui se met en place dans les communautés locales par ou autour des personnes souffrant de troubles psychiques et vivant dans les milieux dits «naturels». Les données sur lesquelles s'appuie cet article ont été cueillies auprès de femmes et d'hommes ayant connu un nombre varié d'hospitalisations psychiatriques depuis plusieurs années et qui sont largement, de ce fait, sous la tutelle de la loi sur l'incapacité mentale. Il s'agit d'entrevues individuelles et semi-dirigées dont les thèmes abordés cherchent à élucider la trajectoire qui a mené à la psychiatrie, ainsi que l'impact de la psychiatrie sur la vie, l'identité et la trajectoire sociale des personnes concernées. Ce sont des données partielles d'une recherche en cours qui comporte d'autres volets, notamment l'étude des pratiques dans les organismes de type communautaire qui desservent les psychiatrisés. L'objectif général de la recherche est de contribuer au renouvellement et à l'élargissement des modèles de pratiques dans le champ de la santé mentale à partir de l'univers de sens des personnes psychiatrisées et de leurs proches ainsi que de l'expérience des regroupements de type associatif ayant émergé au cours des vingt dernières années de désinstitutionnalisation psychiatrique.

5. Malgré le caractère préliminaire de nos analyses, nous pouvons déjà constater une différence entre les récits des hommes et des femmes au niveau du contenu des histoires et expériences. Mais encore plus frappante est la différence dans le rappel de l'histoire lointaine. Tout se passe comme si la mémoire des femmes, et c'est le cas dans cette recherche par exemple, trouvait plus aisément «les mots pour le dire». On ne peut pas commenter plus avant ce constat si ce n'est pour dire qu'il nous parait indiquer une importante piste de recherche sur ce que d'autres ont nommé la «mémoire sexuée» (Devreux, 1986). Nous remercions Cécile Coderre, de l'Université d'Ottawa, de nous avoir signalé cette expression. 